\title{
Clinical relevance of systemic monocytic-MDSCs in patients with metastatic breast cancer
}

\author{
Caroline Bergenfelz ${ }^{1,2} \oplus \cdot$ Anna Roxă $^{3} \cdot$ Meliha Mehmeti $^{1} \cdot$ Karin Leandersson $^{1} \cdot$ Anna-Maria Larsson $^{3,4}$
}

Received: 31 May 2019 / Accepted: 31 December 2019 / Published online: 10 January 2020

(C) The Author(s) 2020

\begin{abstract}
The overall aim of this prospective study was to delineate the role of monocytic myeloid-derived suppressor cells (MoMDSCs) in patients with metastatic breast cancer (MBC). MDSCs are a heterogeneous group of immunosuppressive cells often enriched in different malignancies which hold prognostic and predictive value for clinical outcomes. Here, we assessed the clinical significance of Mo-MDSCs in 54 patients with de novo or distant recurrent MBC. We show that high levels of Mo-MDSCs significantly correlated with de novo MBC (metastatic disease at initial diagnosis), estrogen receptor (ER) negativity, and liver- and bone metastasis. A trend towards an association between high levels of Mo-MDSCs and survival $(P=0.053)$ was also found in patients with distant recurrent ER-positive MBC. We therefore propose that an increased population of Mo-MDSCs may be related to the metastatic or immunoregulatory switch associated with transition to a more systemic disease. Our data imply that high levels of systemic Mo-MDSCs represent patients with more aggressive disease and worse outcome.
\end{abstract}

Keywords Breast cancer · Metastasis · Mo-MDSCs · Survival $\cdot$ Estrogen receptor

$\begin{array}{ll}\text { Abbreviations } \\ \text { AUC } & \text { Area under the curve } \\ \text { CTC } & \text { Circulating tumor cells } \\ \text { ECOG } & \text { Eastern Cooperative Oncology Group } \\ \text { ER } & \begin{array}{l}\text { Estrogen receptor } \\ \text { G-MDSCs }\end{array} \\ \begin{array}{l}\text { Granulocytic myeloid-derived suppressor } \\ \text { cells }\end{array} \\ \text { HER2 } & \text { Human epidermal growth factor receptor } 2 \\ \text { MBC } & \text { Metastatic breast cancer } \\ \text { MDSCs } & \text { Myeloid-derived suppressor cells }\end{array}$

Electronic supplementary material The online version of this article (https://doi.org/10.1007/s00262-019-02472-z) contains supplementary material, which is available to authorized users.

Caroline Bergenfelz

caroline.bergenfelz@med.lu.se

1 Department of Translational Medicine, Cancer Immunology, Lund University, 21428 Malmö, Sweden

2 Department of Translational Medicine, Experimental Infection Medicine, Lund University, Inga Marie Nilssons gata 53, 21428 Malmö, Sweden

3 Department of Clinical Sciences Lund, Division of Oncology and Pathology, Lund University, Lund, Sweden

4 Department of Hematology, Oncology and Radiation Physics, Skåne University Hospital, Lund, Sweden

\begin{tabular}{|c|c|}
\hline MET & Metastasis \\
\hline MFI & Metastasis-free interval \\
\hline Mo-MDSCs & $\begin{array}{l}\text { Monocytic myeloid-derived suppressor } \\
\text { cells }\end{array}$ \\
\hline NHG & Nottingham histological grade \\
\hline OS & Overall survival \\
\hline PBMCs & Peripheral blood mononuclear cells \\
\hline PFS & Progression-free survival \\
\hline PR & Progesterone receptor \\
\hline PT & Primary tumor \\
\hline ROC & Receiver operating characteristic \\
\hline
\end{tabular}

Introduction

Breast cancer is the most common form of malignancy in women [1] and although survival has improved, due to enhanced diagnostics and adjuvant therapies, breast cancer can recur after many years $[2,3]$. When there is progression to metastatic breast cancer (MBC), there is in most cases no curable treatment and the 5-year survival rate is merely $20-30 \%[2,4]$.

Over the past decades, the role of the immune system in cancer development and progression has gained increased attention. Although the exact mechanisms are still being 
investigated, it is known that some immune cells are able to recognize and eliminate tumor cells, a mechanism called immunosurveillance [5, 6]. In order for cancer to develop and progress, malignant cells must escape this surveillance and hijack normal physiological processes typically involved in tissue remodeling, angiogenesis, and cell regeneration [6-8]. One mechanism by which tumors can elude immunosurveillance is through myeloid-derived suppressor cells (MDSCs). MDSCs are a heterogeneous group of highly immunosuppressive cells that accumulate during severe pathological conditions such as sepsis, trauma and cancer [9-11]. Physiologically, they function to dampen excessive immune responses and limit or promote repair of tissue damage $[9,10]$. In cancer, however, the net result would be tumor persistence and progression due to the production of immunosuppressive and pro-angiogenic cytokines and inhibition of anti-tumor T cell responses [10, 12]. The role of MDSCs in breast cancer patients remains relatively unexplored.

Depending on cell surface antigen expression, MDSCs have historically been roughly divided into two groups; granulocyticMDSCs (G-MDSCs; CD1 1 b ${ }^{+} \mathrm{CD} 15^{+} \mathrm{CD} 33^{+} \mathrm{Lin}^{-}{ }^{-} \mathrm{LA}-\mathrm{DR}^{\text {low/-}}{ }^{-}$) and monocytic-MDSCs (Mo-MDSCs; $\mathrm{CD} 11 \mathrm{~b}^{+} \mathrm{CD} 14^{+} \mathrm{CD} 33^{+}$ HLA-DR $^{\text {low/- Co-receptor }}{ }^{\text {low/- }}$ ) [12]. Generally, MDSCs correlate with tumor progression, angiogenesis and poor prognosis in different cancer forms [13-15]. Accordingly, in breast cancer patients, MDSCs are enriched in the peripheral blood and has been proposed to correlate with clinical stage and metastatic burden [16-19]. In patients with stage IV disease, higher levels of MDSCs have also been associated with reduced overall survival $[19,20]$. However, the surface definition of MDSCs has varied substantially between studies and many do not distinguish between G-MDSCs and Mo-MDSCs. A recent study proposes that G-MDSCs $\left(\mathrm{CD} 11 \mathrm{~b}^{+} \mathrm{CD} 33^{+} \mathrm{HLA}^{-} \mathrm{DR}^{-} \mathrm{CD} 15^{+}\right.$ cells) may correlate with triple negative breast cancer and inversely with complete pathologic response after neo-adjuvant chemotherapy [21]. Historically, Mo-MDSCs have been less studied. Enrichment of circulating Mo-MDSCs has previously been identified in patients with melanoma [22, 23], prostate cancer [24], glioblastoma [25], non-Hodgkin lymphoma [26], and bladder cancer [27]. Depending on the type of malignancy, amongst other factors, the presence of Mo-MDSCs correlated with more active [22] or aggressive disease [26] as well as increased tumor size and grade [27]. Whether Mo-MDSCs may provide prognostic or predictive value in patients with breast cancer is currently unknown.

We were recently first to show that Mo-MDSCs are enriched in the peripheral blood of breast cancer patients, primarily in patients with metastatic disease [28]. Here, we aim to further delineate the role of Mo-MDSCs in patients with MBC. By analyzing the frequency of CD $14{ }^{+}$HLA-DR ${ }^{\text {low/-}}$ Co-receptor ${ }^{\text {low/- }}$ Mo-MDSCs in the peripheral blood of patients with MBC we show that Mo-MDSCs significantly correlate to estrogen receptor
(ER)-negative disease, liver- and bone metastases, and de novo MBC (metastatic disease at initial diagnosis). Furthermore, in patients with distant recurrent MBC, high levels of Mo-MDSCs were associated with more circulating tumor cells (CTCs), more metastatic sites and disease progression at 3 month's radiology assessment. In patients with ER-positive distant recurrent $\mathrm{MBC}$, there was a trend towards an association between high levels of Mo-MDSCs and worse overall survival $(P=0.053)$. Altogether this indicates that Mo-MDSCs may be a potential biomarker related to a more aggressive disease and worse outcome in patients with MBC.

\section{Materials and methods}

\section{Patients and study design}

54 patients, with newly diagnosed MBC, scheduled for first line systemic treatment at Skåne University Hospital in Lund, were enrolled between the years 2011 and 2016. This is a sub-study of the prospective observational CTC-MBC study (ClinicalTrials.gov NCT01322893) and clinical information has previously been published [29]. All blood samples were collected before starting systemic therapy for metastatic disease. 13 patients were, however, on endocrine therapy for early breast cancer at the time of MBC diagnosis (see Supplementary Table 1, where previous and current therapy at $\mathrm{MBC}$ diagnosis are indicated). The median follow-up time was 27 months for patients alive at the last medical visit before follow-up cut-off date 2017-05-31. Inclusion criteria were: MBC diagnosis, age $\geq 18$ years, Eastern Cooperative Oncology Group (ECOG) performance status 0-2, and predicted life expectancy of $>2$ months. Exclusion criteria were: prior systemic therapy for metastatic disease, inability to understand the study information, and other malignant disease in the preceding 5 years. Structured clinical and radiological evaluations were performed every 3 months. Progression was defined as progressive disease (PD), whereas non-progression was defined as stable disease (SD) or partial or complete regression (PR and CR), using modified RECIST 1.1 criteria [30]. Patient, tumor, and treatment data were prospectively collected in structured clinical report forms (CRFs). 22 anonymized healthy blood donors were used as controls.

\section{Flow cytometric analysis of Mo-MDSCs}

Peripheral blood was collected in EDTA-coated tubes at baseline, before starting systemic therapy, and analyzed within $24 \mathrm{~h}$ as previously described [28]. Briefly, the peripheral blood was diluted in phosphate-buffered saline (PBS; 
EDTA/sucrose) and overlaid on Ficoll-Paque Plus (cat no. 17-1440-03, GE Healthcare). The peripheral blood mononuclear cells (PBMCs) were collected, washed once in FACS buffer (PBS; BSA/EDTA), and immediately stained for flow cytometry. Antibodies used were as follows: CD14 clone M5E2 (1:10; cat. no 555397), HLA-DR clone G46-6 (1:50; cat. no. 347403), CD80 clone L307.4 (1:15; cat no. 557227), CD86 clone IT2.2 (1:15; cat no. 555665), CD33 clone WM53 (1:10; cat. no 551378), all from BD Biosciences. Analyses were performed using a FACS Calibur (BD Biosciences) and on gated viable (7AAD-negative) PBMCs. Mo-MDSCs were defined as CD14 ${ }^{+} \mathrm{HLA}-\mathrm{DR}{ }^{\text {low/- }}$ cells, where these cells were also deemed $\mathrm{CD} 33^{+}$and with low expression of the co-receptors CD86 and CD80 compared to $\mathrm{CD} 14^{+} \mathrm{HLA}-\mathrm{DR}^{+/ \text {high }}$ cells as well as being immunosuppressive, as demonstrated by their suppression of $\mathrm{T}$ cell proliferation in an ex vivo assay (for full characterization see Bergenfelz et al. [28]). The immunosuppressive profile of Mo-MDSCs from MBC patients was confirmed in a previous study, where flow cytometric analyses of immune cell populations from the first 23 patients included here also have been published [28].

\section{Detection of CTCs}

Blood samples for detection of circulating tumor cells (CTCs) were collected in $10 \mathrm{~mL}$ CellSave Preservation tubes (Menarini Silicon Biosystem, cat. no 7900005), stored between 15 and $30^{\circ} \mathrm{C}$ and processed within $96 \mathrm{~h}$ of collection. CTC enumeration was performed using the CellSearch system (Menarini Silicon Biosystems) as has been described in detail previously [29, 31, 32]. The established cut-off at 5 CTCs was used for defining low levels ( $<5$ CTCs) versus high levels ( $\geq 5$ CTCs) $[29,31]$.

\section{Statistical analyses}

Comparison of Mo-MDSC levels in patients and healthy controls (HC) was made with non-parametric Mann-Whitney Wilcoxon test (for comparison between two groups) and Kruskal Wallis and Dunn's multiple comparison test (for comparison between more than two groups). Categorical patient and tumor characteristics in relation to Mo-MDSC levels were compared using Pearson's chi-squared test and ordinal data were compared using Pearson's chi-squared test for trend. If expected counts were lower than five in one or more of the cells, Fisher's exact test was used. Survival was calculated and illustrated by the log-rank test and Kaplan-Meier curves. Statistical analysis was performed with IBM SPSS Statistics (version 24.0, IBM) and graphs were made with GraphPad Prism (version 8, GraphPad Software).

\section{Results}

\section{Patient characteristics}

54 patients with newly diagnosed MBC were included in this prospective study (Table 1). The median age at MBC diagnosis was 65 years (range 40-84). 36 patients (67\%) had ER-positive primary tumors, 12 (22\%) had ER-negative primary tumors, and seven (13\%) were HER2-positive (five ER-positive and two ER-negative). 21 patients (39\%) had three or more metastatic sites and 34 patients $(63 \%)$ had visceral metastasis. Bone metastases were most prevalent (42 patients; 78\%), followed by lymph node and lung metastases (23 patients; $43 \%$ and 21 patients; $39 \%$, respectively). 16 patients were diagnosed with liver metastasis (30\%). 12 patients $(22 \%)$ had de novo MBC at initial breast cancer diagnosis, while 42 patients (78\%) in the studied group were diagnosed with distant recurrence. The median metastasisfree interval (MFI) for patients with distant recurrent MBC was 6.4 years (range 1.0-27.2). Patient characteristics are summarized in Table 1.

\section{Mo-MDSCs are enriched in the peripheral blood of a subpopulation of $M B C$ patients}

Peripheral blood was collected from all patients at baseline, before starting systemic treatment for MBC. Circulating MoMDSCs (CD14 ${ }^{+} \mathrm{HLA}^{-D R^{\text {low/-}}}{ }^{-} \mathrm{Co}-$ receptor $^{\text {low/- }}$ cells) in the PBMC fraction were analyzed by flow cytometry. Gating strategies and representative mean fluorescence intensity (MFI) for co-receptors are depicted in Supplementary Fig. 1. The suppressive activity of the monocytes (suppression of $\mathrm{T}$ lymphocytes and increased release of IL-10) has previously been characterized in a study where we analyzed MoMDSCs from the first 23 patients enrolled [28]. In accordance with this study, Mo-MDSCs were significantly enriched in the peripheral blood of a subpopulation of MBC patients (Fig. 1a and Supplementary Fig. 1a). Based on a previously defined cut-off (highest Mo-MDSC value received from healthy controls [28]; 8.29\% of PBMCs), the MBC patients were stratified into groups with "normal" or "high" Mo-MDSC levels. 28 patients displayed normal levels of Mo-MDSCs (range 1.45-8.03\% of PBMCs) and 26 patients had high levels (range $8.47-22.57 \%$ of PBMCs, Fig. 1a). No significant difference with regards to levels of Mo-MDSCs was observed between patients on endocrine therapy or not at the time of MBC diagnosis nor for patients that previously had received adjuvant chemo- or endocrine therapy (Supplementary Table 1).

In order to assess the performance of Mo-MDSCs as a biomarker to distinguish healthy control and $\mathrm{MBC}$, we 
Table 1 Clinicopathological variables in all patients, stratified by levels of Mo-MDSCs

\begin{tabular}{|c|c|c|c|c|}
\hline Clinicopathological variable & $\begin{array}{l}\text { All patients } \\
n=54\end{array}$ & $\begin{array}{l}\text { Normal Mo-MDSCs } \\
n=28(\%)\end{array}$ & $\begin{array}{l}\text { High Mo-MDSCs } \\
n=26(\%)\end{array}$ & $P$ value \\
\hline \multicolumn{5}{|l|}{ Age (years) } \\
\hline$<65$ & 27 & $13(46)$ & $14(54)$ & \multirow[t]{2}{*}{$0.59^{\mathrm{a}}$} \\
\hline$\geq 65$ & 27 & $15(54)$ & $12(46)$ & \\
\hline \multicolumn{5}{|l|}{ Baseline $^{c}$ ECOG $^{d}$} \\
\hline 0 & 35 & $20(71)$ & $15(60)$ & \multirow[t]{4}{*}{$0.71^{\mathrm{b}}$} \\
\hline 1 & 6 & $3(11)$ & $3(12)$ & \\
\hline 2 & 12 & $5(18)$ & $7(28)$ & \\
\hline Unknown & 1 & 0 & 1 & \\
\hline \multicolumn{5}{|l|}{ Tumor type } \\
\hline Ductal & 40 & $22(82)$ & $18(72)$ & \multirow[t]{4}{*}{$0.45^{\mathrm{b}}$} \\
\hline Lobular & 9 & $3(11)$ & $6(24)$ & \\
\hline Other & 3 & $2(7)$ & $1(4)$ & \\
\hline Unknown & 2 & 1 & 1 & \\
\hline \multicolumn{5}{|l|}{ PT NHG } \\
\hline I & 4 & $3(13)$ & $1(8)$ & \multirow[t]{4}{*}{$0.88^{b}$} \\
\hline II & 23 & $14(58)$ & $9(69)$ & \\
\hline III & 10 & $7(29)$ & $3(23)$ & \\
\hline Unknown & 17 & 4 & 13 & \\
\hline \multicolumn{5}{|l|}{ PT tumor size } \\
\hline $\mathrm{T} 1$ & 20 & $13(50)$ & $7(32)$ & \multirow[t]{5}{*}{$0.38^{\mathrm{b}}$} \\
\hline $\mathrm{T} 2$ & 14 & $8(31)$ & $6(27)$ & \\
\hline $\mathrm{T} 3$ & 7 & $2(8)$ & $5(23)$ & \\
\hline $\mathrm{T} 4$ & 7 & $3(11)$ & $4(18)$ & \\
\hline Unknown & 6 & 2 & 4 & \\
\hline \multicolumn{5}{|l|}{ PT node status } \\
\hline Negative & 15 & $11(42)$ & $4(20)$ & \multirow[t]{3}{*}{$0.11^{\mathrm{a}}$} \\
\hline Positive & 31 & $15(58)$ & $16(80)$ & \\
\hline Unknown & 8 & 2 & 6 & \\
\hline \multicolumn{5}{|l|}{ PT hormone receptor status } \\
\hline ER-negative & 12 & $3(12)$ & $9(41)$ & \multirow[t]{2}{*}{$0.02^{\mathrm{a}}$} \\
\hline ER-positive & 36 & $23(88)$ & $13(59)$ & \\
\hline Unknown & 6 & 2 & 4 & \multirow{4}{*}{$0.13^{\mathrm{a}}$} \\
\hline PR-negative & 19 & $8(31)$ & $11(52)$ & \\
\hline PR-positive & 28 & $18(69)$ & $10(48)$ & \\
\hline Unknown & 7 & 2 & 5 & \\
\hline \multicolumn{5}{|l|}{ PT HER2 status } \\
\hline HER2-negative & 32 & $18(86)$ & $14(78)$ & \multirow[t]{3}{*}{$0.68^{b}$} \\
\hline HER2-positive & 7 & $3(14)$ & $4(22)$ & \\
\hline Unknown & 15 & 7 & 8 & \\
\hline \multicolumn{5}{|l|}{ MET hormone receptor status } \\
\hline ER-negative & 7 & $2(8)$ & $5(23)$ & \multirow[t]{2}{*}{$0.23^{\mathrm{b}}$} \\
\hline ER-positive & 40 & $23(92)$ & $17(77)$ & \\
\hline Unknown & 7 & 3 & 4 & \multirow{4}{*}{$0.52^{\mathrm{a}}$} \\
\hline PR-negative & 27 & $13(54)$ & $14(64)$ & \\
\hline PR-positive & 19 & $11(46)$ & $8(36)$ & \\
\hline Unknown & 8 & 4 & 4 & \\
\hline \multicolumn{5}{|l|}{ MET HER2 status } \\
\hline HER2-negative & 39 & $19(83)$ & $20(95)$ & $0.35^{\mathrm{b}}$ \\
\hline HER2-positive & 5 & $4(17)$ & $1(5)$ & \\
\hline Unknown & 10 & 5 & 5 & \\
\hline
\end{tabular}


Table 1 (continued)

\begin{tabular}{|c|c|c|c|c|}
\hline Clinicopathological variable & $\begin{array}{l}\text { All patients } \\
n=54\end{array}$ & $\begin{array}{l}\text { Normal Mo-MDSCs } \\
n=28(\%)\end{array}$ & $\begin{array}{l}\text { High Mo-MDSCs } \\
n=26(\%)\end{array}$ & $P$ value \\
\hline \multicolumn{5}{|l|}{ Metastatic sites, $n$} \\
\hline$<3$ & 33 & $20(71)$ & $13(50)$ & \multirow[t]{2}{*}{$0.11^{\mathrm{a}}$} \\
\hline$\geq 3$ & 21 & $8(29)$ & $13(50)$ & \\
\hline \multicolumn{5}{|l|}{ Metastatic sites, localization } \\
\hline \multicolumn{5}{|l|}{ Lymph node } \\
\hline Negative & 31 & $18(64)$ & $13(50)$ & \multirow[t]{2}{*}{$0.29^{\mathrm{a}}$} \\
\hline Positive & 23 & $10(36)$ & $13(50)$ & \\
\hline \multicolumn{5}{|l|}{ Lung } \\
\hline Negative & 33 & $16(57)$ & $17(65)$ & \multirow[t]{2}{*}{$0.54^{\mathrm{a}}$} \\
\hline Positive & 21 & $12(43)$ & $9(35)$ & \\
\hline \multicolumn{5}{|l|}{ Liver } \\
\hline Negative & 38 & $23(82)$ & $15(58)$ & \multirow[t]{2}{*}{$<0.05^{\mathrm{a}}$} \\
\hline Positive & 16 & $5(18)$ & $11(42)$ & \\
\hline \multicolumn{5}{|l|}{ Bone } \\
\hline Negative & 12 & $10(36)$ & $2(8)$ & \multirow[t]{2}{*}{$0.01^{\mathrm{a}}$} \\
\hline Positive & 42 & $18(64)$ & $24(92)$ & \\
\hline \multicolumn{5}{|l|}{ Visceral $^{\mathrm{e}}$} \\
\hline Non-visceral & 20 & $11(39)$ & $9(35)$ & \multirow[t]{2}{*}{$0.72^{\mathrm{a}}$} \\
\hline Visceral & 34 & $17(61)$ & $17(65)$ & \\
\hline \multicolumn{5}{|l|}{ Bone only } \\
\hline Not bone-only & 43 & $22(79)$ & $21(81)$ & \multirow[t]{2}{*}{$0.84^{\mathrm{a}}$} \\
\hline Bone-only & 11 & $6(21)$ & $5(19)$ & \\
\hline \multicolumn{5}{|l|}{ Progression at 3 mo evaluation } \\
\hline Non-progression & 39 & $23(92)$ & $16(73)$ & \multirow[t]{3}{*}{$0.12^{\mathrm{b}}$} \\
\hline Progression & 8 & $2(8)$ & $6(27)$ & \\
\hline Unknown & 7 & 3 & 4 & \\
\hline \multicolumn{5}{|l|}{ Type of MBC } \\
\hline De novo ${ }^{f}$ & 12 & $2(7)$ & $10(38)$ & \multirow[t]{2}{*}{$0.006^{\mathrm{a}}$} \\
\hline Distant recurrent ${ }^{\mathrm{g}}$ & 42 & $26(93)$ & $16(62)$ & \\
\hline \multicolumn{5}{|l|}{ CTC at baseline $\mathrm{c}^{\mathrm{c}}$} \\
\hline$<5$ & 25 & $16(59)$ & $9(35)$ & \multirow[t]{3}{*}{$0.07^{\mathrm{a}}$} \\
\hline$\geq 5$ & 28 & $11(41)$ & $17(65)$ & \\
\hline Unknown & 1 & 1 & 0 & \\
\hline
\end{tabular}

Number of patients and percentage (\%) distribution of patients indicated for each variable

Mo-MDSCs monocytic myeloid-derived suppressor cells, $M B C$ metastatic breast cancer, ECOG Eastern Cooperative Oncology Group, $P T$ primary tumor, $N H G$ Nottingham histological grade, $E R$ estrogen receptor, $P R$ progesterone receptor, $H E R 2$ human epidermal growth factor receptor 2, MET metastasis, $C T C$ circulating tumor cells

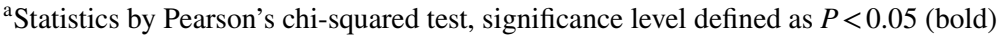

${ }^{\text {b}}$ Statistics by Fisher's exact test. Used when presence of expected values less than 5 . Significance level defined as $P<0.05$ (bold)

${ }^{\mathrm{c}}$ Baseline defined as a time point before starting first line systemic MBC treatment

${ }^{\mathrm{d}}$ ECOG denotes the performance status used in clinical practice in Sweden

${ }^{\mathrm{e}}$ Visceral metastasis defined as lung, liver, brain, peritoneal, and/or pleural involvement

${ }^{\mathrm{f}}$ De novo MBC defined as MBC at initial breast cancer diagnosis

${ }^{g}$ Distant recurrent MBC defined as MBC diagnosis after $>0$ years after primary diagnosis

used receiver operating characteristic (ROC) analyses. The frequency of Mo-MDSCs achieved an area under the curve (AUC) value of $0.74(95 \% \mathrm{CI} 0.628-0.845, P=0.0013)$ to distinguish MBC patients from healthy controls (Fig. 1b). This indicates that the levels of Mo-MDSCs may function as a biomarker in MBC. 

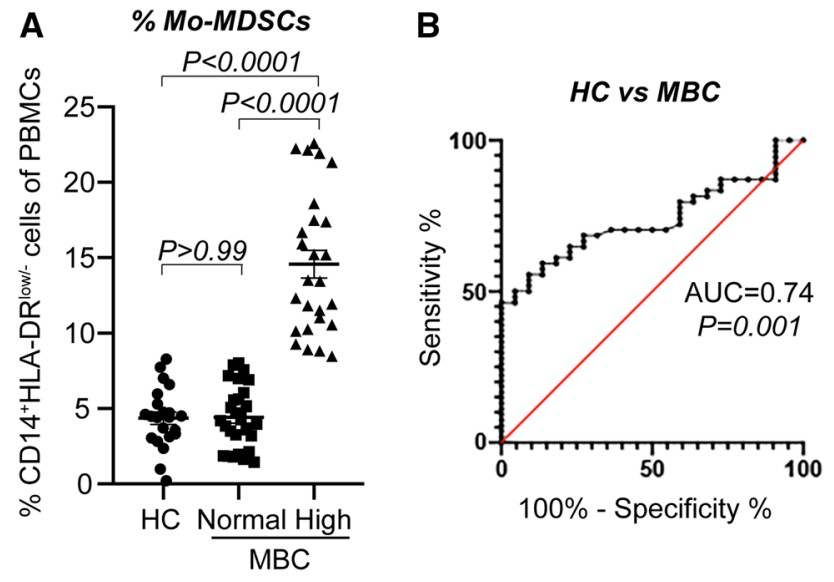

Fig. 1 Mo-MDSCs are enriched in the peripheral blood of MBC patients. Peripheral blood was collected from 54 patients with metastatic breast cancer (MBC) and 22 healthy controls (HC). Percentage of CD14 ${ }^{+} \mathrm{HLA}-\mathrm{DR}{ }^{\text {low/- }}$ Mo-MDSCs was assessed by flow cytometry. a Median percentage of Mo-MDSC in $\mathrm{HC}$ and MBC patients with normal $(n=28)$ and high $(n=26)$ levels of Mo-MDSCs. Cut-off level between normal and high levels of Mo-MDSCs was set to the highest value of Mo-MDSCs in HCs (8.29\% of PBMCs), as described in [28]. Error bars; SEM. Exact $P$ values, by Kruskal Wallis with Dunn's multiple comparison test, are indicated. b Receiver operating characteristic (ROC) curve of percentage Mo-MDSCs in healthy controls (HC, $n=22)$ and MBC patients $(n=54)$. Area under the curve (AUC) and significance are indicated

\section{Mo-MDSCs correlate with ER-negativity and liver or bone metastases}

In order to determine the clinical significance of MoMDSCs, clinicopathological features were compared in patients with normal versus high levels of Mo-MDSCs (Table 1). There was no significant difference between patients with normal- and high levels of Mo-MDSCs regarding age, performance status (Eastern Cooperative Oncology Group, ECOG), tumor type, size or histologic grade (Nottingham histological grade, NHG; Table 1). Significantly higher frequency of ER-negative primary tumors was seen among the patients with high levels of Mo-MDSCs compared to patients with normal levels $(41 \%$ and $12 \%$ of patients, respectively; Table 1). Furthermore, significantly more liver metastases were seen in the group of patients with high levels of Mo-MDSCs compared to the group with normal levels of Mo-MDSCs (42\% and 18\% of patients, respectively; Table 1). Similarly, more patients with high levels of Mo-MDSCs had bone metastases compared to patients with normal Mo-MDSC levels (92\% and 64\%, respectively; Table 1 and Fig. 2a). Tendencies were also seen in correlations between high levels of Mo-MDSCs and metastatic burden ( $\geq 3$ metastatic sites), number of circulating tumor cells (CTCs), and progression at three months' radiology evaluation, where patients with high levels of Mo-MDSCs
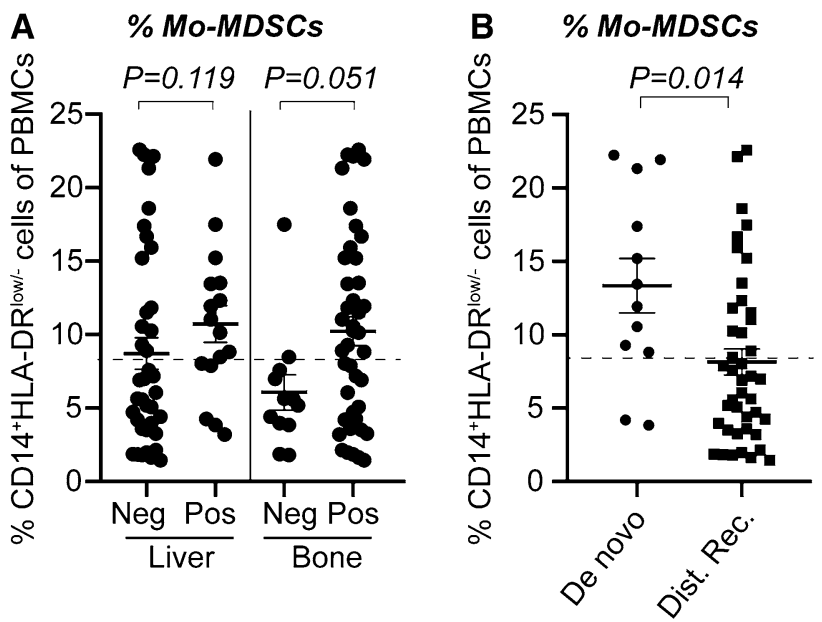

Fig. 2 Percentages of Mo-MDSCs in patients divided by clinicopathological features. CD14 ${ }^{+}$HLA-DR ${ }^{\text {low/- }}$ Mo-MDSCs in peripheral blood was assessed by flow cytometry and stratified according to clinicopathological features (see Table 1). Median percentage of Mo-MDSCs as divided by (a) metastatic site or (b) according to de novo or distant recurrent MBC. Error bars; SEM. Exact $P$ values, by Mann-Whitney Wilcoxon, are indicated

tended to have more metastatic sites, higher CTC levels, and more patients had progression at first evaluation (Table 1).

\section{High level of Mo-MDSCs is associated with de novo MBC}

Interestingly, patients with de novo MBC were overrepresented in the group with high levels of Mo-MDSCs (Table 1). More than $80 \%$ of patients with de novo MBC had high Mo-MDSC levels, as compared with $38 \%$ of patients with distant recurrent MBC $(P=0.006$; Tables 1 , 2; Fig. 2b). This dramatic difference urged us to clarify the role of Mo-MDSCs in the de novo MBC patients versus the distant recurrent $\mathrm{MBC}$ patients. No differences were seen between the two patient groups in terms of age, performance status, and several other important clinicopathological variables (Tables 1, 2). In the group with de novo MBC, all ten patients with high levels of Mo-MDSCs had bone metastasis (Table 2). The group with distant recurrent MBC patients with high levels of Mo-MDSCs tended to have more liver metastasis than patients with low Mo-MDSCs $(P=0.07$; Table 2). Due to few patients in the de novo and distant recurrent MBC groups, differences were not statistically significant.

\section{Associations of circulating Mo-MDSCs and outcome}

To evaluate the prognostic impact of Mo-MDSCs in all patients with MBC, we used Kaplan-Meier curves to compare progression-free survival (PFS) and overall survival 
Table 2 Clinicopathological variables in de novo MBC versus distant recurrent MBC, stratified by levels of Mo-MDSCs

\begin{tabular}{|c|c|c|c|c|c|}
\hline \multirow[t]{2}{*}{ Clinicopathological variable } & \multicolumn{2}{|l|}{ De novo $\mathrm{MBC}^{\mathrm{a}}$} & \multicolumn{2}{|c|}{ Distant recurrent $\mathrm{MBC}^{\mathrm{b}}$} & \multirow[t]{2}{*}{$P$ value $^{\mathrm{c}}$} \\
\hline & $\begin{array}{l}\text { Normal levels } \\
n=2(\%)\end{array}$ & $\begin{array}{l}\text { High levels } \\
n=10(\%)\end{array}$ & $\begin{array}{l}\text { Normal levels } \\
n=26(\%)\end{array}$ & $\begin{array}{l}\text { High levels } \\
n=16(\%)\end{array}$ & \\
\hline \multicolumn{6}{|l|}{ Age (years) } \\
\hline$<65$ & $1(50)$ & $7(70)$ & $12(46)$ & $7(44)$ & \multirow[t]{2}{*}{1.0} \\
\hline$\geq 65$ & $1(50)$ & $3(30)$ & $14(54)$ & $9(56)$ & \\
\hline \multicolumn{6}{|l|}{ Baseline $^{\mathrm{d}} \mathrm{ECOG}^{\mathrm{e}}$} \\
\hline 0 & $1(50)$ & $6(60)$ & $19(73)$ & $9(60)$ & \multirow[t]{4}{*}{0.70} \\
\hline 1 & $0(0)$ & $0(0)$ & $3(12)$ & $3(20)$ & \\
\hline 2 & $1(50)$ & $4(40)$ & $4(15)$ & $3(20)$ & \\
\hline Unknown & 0 & 0 & 0 & 1 & \\
\hline \multicolumn{6}{|l|}{ Tumor type } \\
\hline Ductal & $2(100)$ & $8(89)$ & $20(80)$ & $10(63)$ & \multirow[t]{4}{*}{0.31} \\
\hline Lobular & $0(0)$ & $1(11)$ & $3(12)$ & $5(31)$ & \\
\hline Other & $0(0)$ & $0(0)$ & $2(8)$ & $1(6)$ & \\
\hline Unknown & 0 & 1 & 1 & 0 & \\
\hline \multicolumn{6}{|l|}{ PT NHG } \\
\hline I & - & - & $3(13)$ & $1(8)$ & \multirow[t]{4}{*}{0.88} \\
\hline II & - & - & $14(58)$ & $9(69)$ & \\
\hline III & - & - & $7(29)$ & $3(23)$ & \\
\hline Unknown & & & 4 & 13 & \\
\hline \multicolumn{6}{|l|}{ PT tumor size } \\
\hline $\mathrm{T} 1$ & $0(0)$ & $0(0)$ & $13(50)$ & $7(46)$ & \multirow[t]{5}{*}{0.30} \\
\hline $\mathrm{T} 2$ & $0(0)$ & $2(29)$ & $8(31)$ & $4(27)$ & \\
\hline $\mathrm{T} 3$ & $0(0)$ & $1(14)$ & $2(8)$ & $4(27)$ & \\
\hline $\mathrm{T} 4$ & $0(0)$ & $4(57)$ & $3(11)$ & $0(0)$ & \\
\hline Unknown & 2 & 3 & 0 & 1 & \\
\hline \multicolumn{6}{|l|}{ PT node status } \\
\hline Negative & $0(0)$ & $0(0)$ & $11(42)$ & $4(25)$ & \multirow[t]{3}{*}{0.33} \\
\hline Positive & $0(0)$ & $4(100)$ & $15(58)$ & $12(75)$ & \\
\hline Unknown & 2 & 6 & 0 & 0 & \\
\hline \multicolumn{6}{|l|}{ PT hormone receptor status } \\
\hline ER-negative & $0(0)$ & $4(44)$ & $3(12)$ & $5(38)$ & \multirow[t]{3}{*}{0.09} \\
\hline ER-positive & $1(100)$ & $5(56)$ & $22(88)$ & $8(62)$ & \\
\hline Unknown & 1 & 1 & 1 & 3 & \\
\hline PR-negative & $0(0)$ & $4(44)$ & $8(32)$ & $7(58)$ & \multirow[t]{3}{*}{0.16} \\
\hline PR-positive & $1(100)$ & $5(56)$ & $17(68)$ & $5(42)$ & \\
\hline Unknown & 1 & 1 & 1 & 4 & \\
\hline \multicolumn{6}{|l|}{ PT HER2 status } \\
\hline HER2-negative & $0(0)$ & $6(67)$ & $18(90)$ & $8(89)$ & \multirow[t]{3}{*}{1.0} \\
\hline HER2-positive & $1(100)$ & $3(33)$ & $2(10)$ & $1(11)$ & \\
\hline Unknown & 1 & 1 & 6 & 7 & \\
\hline \multicolumn{6}{|l|}{ MET hormone receptor status } \\
\hline ER-negative & $1(50)$ & $2(33)$ & $1(4)$ & $3(19)$ & 0.29 \\
\hline ER-positive & $1(50)$ & $4(67)$ & $22(96)$ & $13(81)$ & \\
\hline Unknown & 0 & 4 & 3 & 0 & \\
\hline PR-negative & $1(50)$ & $3(50)$ & $12(55)$ & $11(69)$ & 0.51 \\
\hline PR-positive & $1(50)$ & $3(50)$ & $10(45)$ & $5(31)$ & \\
\hline Unknown & 0 & 4 & 4 & 0 & \\
\hline
\end{tabular}


Table 2 (continued)

\begin{tabular}{|c|c|c|c|c|c|}
\hline \multirow[t]{2}{*}{ Clinicopathological variable } & \multicolumn{2}{|l|}{ De novo $\mathrm{MBC}^{\mathrm{a}}$} & \multicolumn{2}{|c|}{ Distant recurrent $\mathrm{MBC}^{\mathrm{b}}$} & \multirow[t]{2}{*}{$P$ value } \\
\hline & $\begin{array}{l}\text { Normal levels } \\
n=2(\%)\end{array}$ & $\begin{array}{l}\text { High levels } \\
n=10(\%)\end{array}$ & $\begin{array}{l}\text { Normal levels } \\
n=26(\%)\end{array}$ & $\begin{array}{l}\text { High levels } \\
n=16(\%)\end{array}$ & \\
\hline \multicolumn{6}{|l|}{ MET HER2 status } \\
\hline HER2-negative & $1(50)$ & $6(100)$ & $18(86)$ & $14(93)$ & 0.63 \\
\hline HER2-positive & $1(50)$ & $0(0)$ & $3(14)$ & $1(7)$ & \\
\hline Unknown & 0 & 4 & 5 & 1 & \\
\hline \multicolumn{6}{|l|}{ Metastatic sites, $n$} \\
\hline$<3$ & $1(50)$ & $4(40)$ & $19(73)$ & $9(56)$ & 0.32 \\
\hline$\geq 3$ & $1(50)$ & $6(60)$ & $7(27)$ & $7(44)$ & \\
\hline \multicolumn{6}{|l|}{ Metastatic sites, localization } \\
\hline \multicolumn{6}{|l|}{ Lymph node } \\
\hline Negative & $0(0)$ & $2(20)$ & 18 (69) & $11(69)$ & 1 \\
\hline Positive & $2(100)$ & $8(80)$ & $8(31)$ & $5(31)$ & \\
\hline \multicolumn{6}{|l|}{ Lung } \\
\hline Negative & $1(50)$ & $7(70)$ & $15(58)$ & $10(62)$ & 1 \\
\hline Positive & $1(50)$ & $3(30)$ & $11(42)$ & $6(38)$ & \\
\hline \multicolumn{6}{|l|}{ Liver } \\
\hline Negative & $1(50)$ & $6(60)$ & $22(85)$ & $9(56)$ & 0.07 \\
\hline Positive & $1(50)$ & $4(40)$ & $4(15)$ & $7(44)$ & \\
\hline \multicolumn{6}{|l|}{ Bone } \\
\hline Negative & $1(50)$ & $0(0)$ & $9(35)$ & $2(13)$ & 0.16 \\
\hline Positive & $1(50)$ & $10(100)$ & $17(65)$ & 14 (87) & \\
\hline \multicolumn{6}{|l|}{ Visceral $^{\mathrm{f}}$} \\
\hline Non-visceral & $0(0)$ & $4(40)$ & $11(42)$ & $5(31)$ & 0.53 \\
\hline Visceral & $2(100)$ & $6(60)$ & $15(58)$ & $11(69)$ & \\
\hline \multicolumn{6}{|l|}{ Bone only } \\
\hline Not bone-only & $2(100)$ & $9(90)$ & $20(77)$ & $12(75)$ & 1 \\
\hline Bone-only & $0(0)$ & $1(10)$ & $6(23)$ & $4(25)$ & \\
\hline \multicolumn{6}{|c|}{ Progression at 3 months' evaluation } \\
\hline Non-progression & $1(100)$ & $7(88)$ & $22(92)$ & $9(64)$ & 0.08 \\
\hline Progression & $0(0)$ & $1(12)$ & $2(8)$ & $5(36)$ & \\
\hline Unknown & 1 & 2 & 2 & 2 & \\
\hline \multicolumn{6}{|l|}{ CTC at baseline ${ }^{\mathrm{d}}$} \\
\hline$<5$ & $1(100)$ & $4(40)$ & $15(58)$ & $5(31)$ & 0.12 \\
\hline$\geq 5$ & $0(0)$ & $6(60)$ & $11(42)$ & $11(69)$ & \\
\hline Unknown & 1 & 0 & 0 & 0 & \\
\hline
\end{tabular}

Number of patients and percentage $(\%)$ distribution of patients indicated for each variable

$M B C$ metastatic breast cancer, Mo-MDSCs monocytic myeloid-derived suppressor cells, ECOG Eastern Cooperative Oncology Group, $P T$ primary tumor, $E R$ estrogen receptor, $P R$ progesterone receptor, $H E R 2$ human epidermal growth factor receptor 2, MET metastasis, CTC circulating tumor cells

${ }^{a}$ De novo MBC defined as MBC at initial breast cancer diagnosis, $n$ too small for statistical analysis and no $P$ values are listed

${ }^{\mathrm{b}}$ Distant recurrent $\mathrm{MBC}$ defined as $\mathrm{MBC}$ diagnosis after $>0$ years after primary diagnosis

${ }^{\mathrm{c}}$ Statistics by Fisher's exact test. Significance level defined as $P<0.05$ (bold)

${ }^{\mathrm{d}}$ Baseline defined as a time point before starting first line systemic MBC treatment

${ }^{e}$ ECOG denotes the performance status used in clinical practice in Sweden

${ }^{\mathrm{f}}$ Visceral metastasis is defined as lung, liver, brain, peritoneal, and/or pleural involvement 
A

Progression-free survival

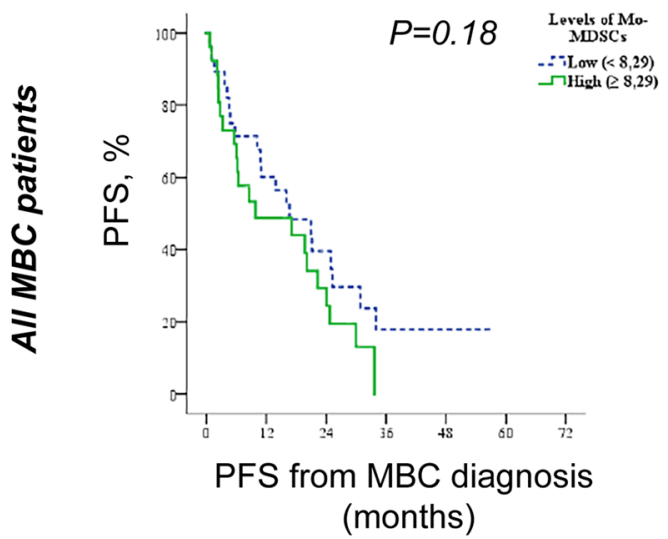

No. at risk

Normal (<8.29) $28 \quad 16 \quad 8 \quad 3 \quad 2$

High ( $\geq 8.29) \quad 26 \quad 11 \quad 6 \quad 0 \quad 0$

C

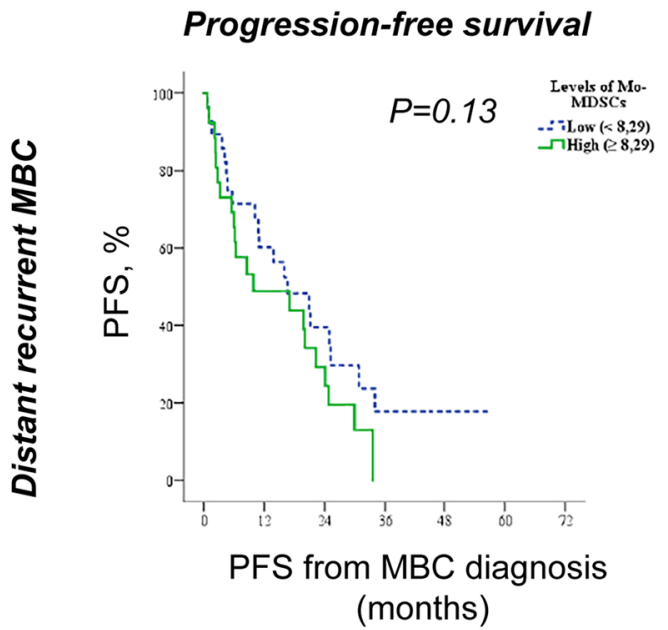

No. at risk

Normal $(<8.29) \quad 26 \quad 16 \quad 8 \quad 3 \quad 2$

High $(\geq 8.29) \quad 16 \quad 6 \quad 4 \quad 00$

Fig. 3 Mo-MDSC levels and associations with survival. KaplanMeier curves of progression-free survival (PFS) and overall survival (OS) according to Mo-MDSC levels in all MBC patients $(n=54$,

(OS) in patients with normal and high Mo-MDSC levels. Patients with normal levels of Mo-MDSCs tended to have improved PFS compared to patients with high levels of Mo-MDSCs (median PFS; 16.6 months 95\% CI 5.8-27.5 and 9.9 months $95 \%$ CI $0-25.1$, respectively, $P=0.18$; Fig. 3a). Similarly, OS tended to be better for patients with normal compared to high Mo-MDSC levels (median OS; 43.2 months $95 \%$ CI $12.3-74.1$ and 40.3 months $95 \%$ CI $7.9-72.8$, respectively, $P=0.24$; Fig. 3b). This was specific for Mo-MDSCs as the levels of monocytes (all CD14 ${ }^{+}$cells, divided by median value $12.0 \%$ of PBMCs) did not relate
B

Overall survival

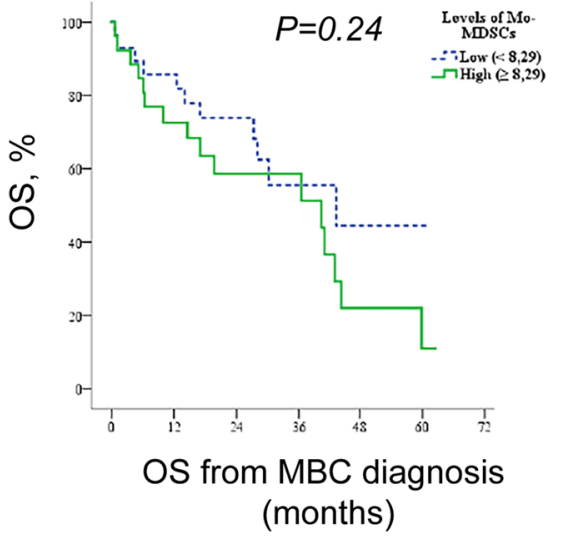

No. at risk

Normal $(<8.29) \quad 28 \quad 23 \quad 15 \quad 6 \quad 3$

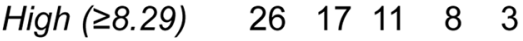

D

Overall survival

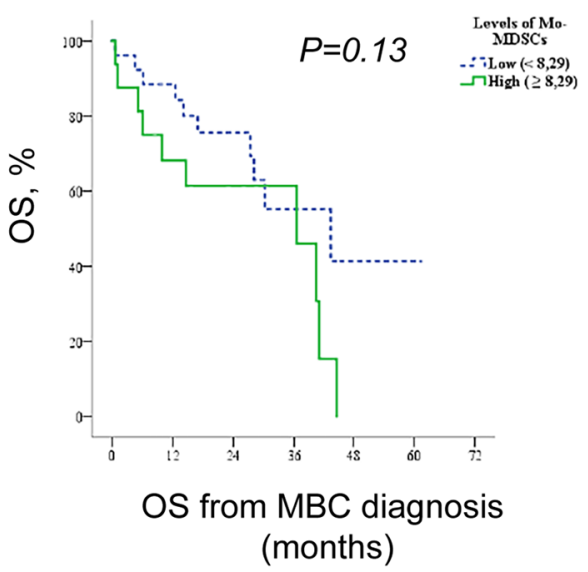

No. at risk

Normal (<8.29) $26 \quad 22 \quad 14 \quad 5 \quad 3$

$\operatorname{High}(\geq 8.29) \quad 16 \quad 10 \quad 7 \quad 4 \quad 0$

a-b) or in MBC patients with distant recurrence $(n=42, \mathbf{c}-\mathbf{d})$. Statistics by Log-rank test

to either PFS (Supplementary Fig. 2a) or OS (Supplementary Fig. 2b). Neither did the monocyte-levels (all CD14 ${ }^{+}$ cells) correlate with any of the described clinicopathological parameters (data not shown).

As the role of Mo-MDSCs could be different in patients with de novo and distant recurrent MBC, we also assessed the impact of Mo-MDSCs in patients with distant recurrent MBC only. Similar tendencies regarding PFS (Fig. 3c) and OS (Fig. 3d) were observed. Median PFS of patients with distant recurrent $\mathrm{MBC}$ displaying normal and high Mo-MDSC levels were 20.9 months (95\% CI 13.5-28.3) 
and 8.5 months (95\% CI 2.0-15.1), respectively $(P=0.13$; Fig. 3C), and median OS of patients with distant recurrent MBC displaying normal and high Mo-MDSC levels were 43.2 months (95\% CI 15.5-71.0) and 36.5 months (95\% CI 9.8-63.1), respectively ( $P=0.13$, Fig. 3d). This is in accordance with the correlation of high levels of Mo-MDSCs and disease progression at first radiology evaluation after 3 months of treatment $(P=0.08$; Table 2$)$ in this patient group.

Finally, in subgroup analysis of patients with distant recurrent MBC, we found that high Mo-MDSC levels are more strongly associated with worse PFS and OS in patients with ER-positive tumors (Fig. 4a-b) than
ER-negative tumors (Fig. 4c-d). In patients with ERpositive primary tumors and high Mo-MDSC levels, median PFS was 6.2 months (95\% CI 0-15.3) compared to 24.9 months (95\% CI 17.5-32.3) for patients with low Mo-MDSC levels $(P=0.078$; Fig. 4a). For OS similar associations were seen with worse survival in patients with high Mo-MDSC levels $(P=0.053$; Fig. 4b). Also, when analyzing Mo-MDSC levels and possible correlations to survival in all patients (de novo and distant recurrent MBC) stratified for tumor ER status, there was a trend towards impaired OS in patients with high Mo-MDSC levels in the ER-positive tumor group $(P=0.06)$, whereas in the group of patients with ER-negative tumors there was
A

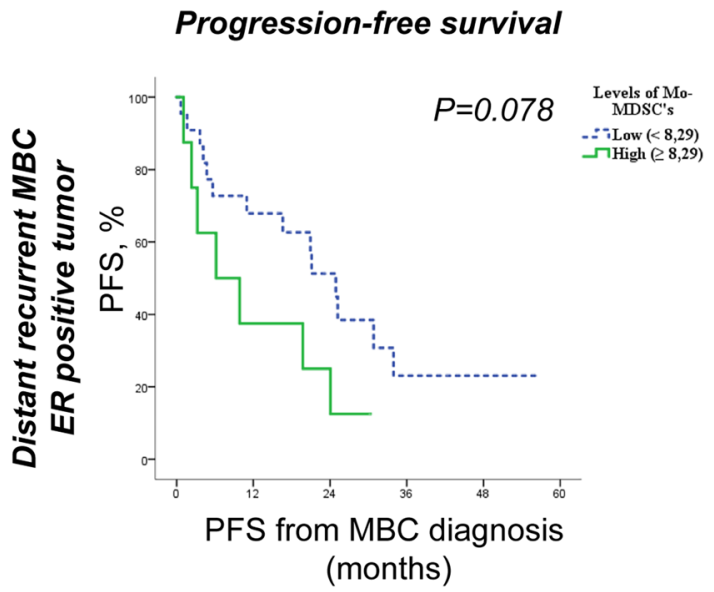

B Overall survival

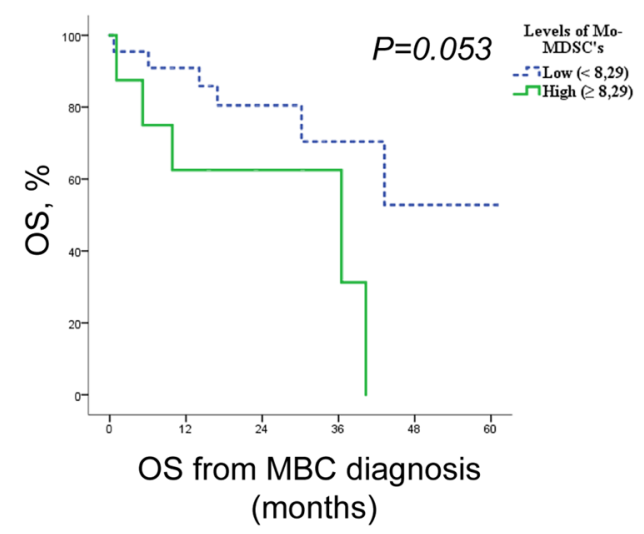

No. at risk

Normal $(<8.29) \quad 22 \quad 14 \quad 8 \quad 3 \quad 2$

\section{No. at risk}

Normal $(<8.29) \quad 22 \quad 19 \quad 12 \quad 5 \quad 3$

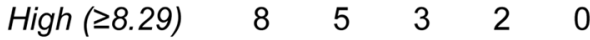

C

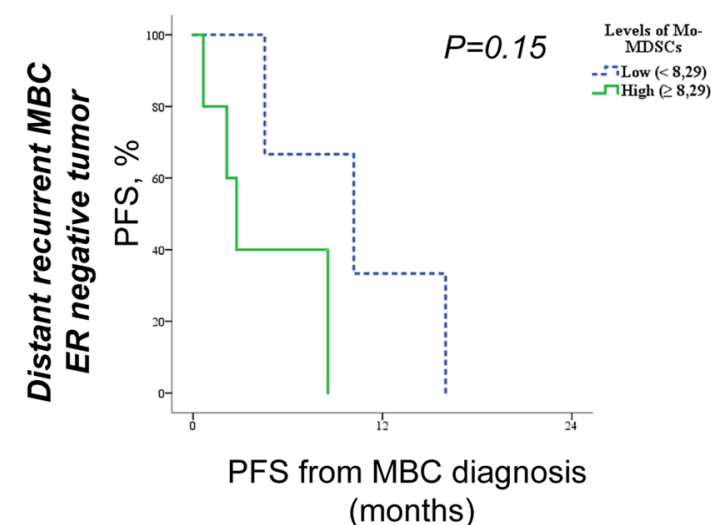

No. at risk

Normal $(<8.29) \quad 3$

High ( $\geq 8.29)$
Progression-free survival

(months)
Fig. 4 Mo-MDSC levels and associations with survival in distant recurrent MBC patients stratified for tumor ER status. Kaplan-Meier curves of progression-free survival (PFS) and overall survival (OS) in

\section{Overall survival}

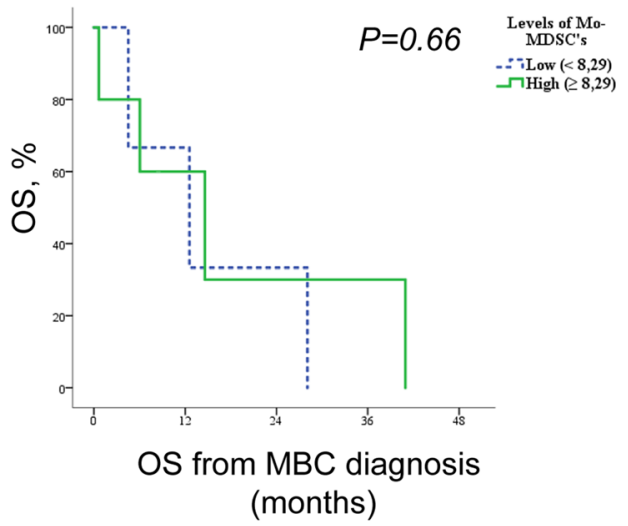

No. at risk

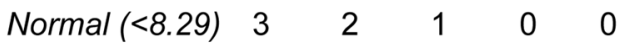

$\operatorname{High}(\geq 8.29) \quad 5 \quad 2 \quad 1 \quad 1 \quad 0$ relation to Mo-MDSC levels in MBC patients with ER-positive primary tumors $(n=30, \mathbf{a}-\mathbf{b})$ or in MBC patients with ER-negative primary tumors $(n=8, \mathbf{c}-\mathbf{d})$. Statistics by Log-rank test 
no difference in OS in relation to Mo-MDSC levels (Supplementary Fig. 3).

\section{Discussion}

In the present study we evaluated the role of systemic MoMDSCs in patients with newly diagnosed MBC. A high level of Mo-MDSCs was significantly correlated with ERnegative primary tumors, as well as with liver- and bone metastases, which is in accordance with our previous study, comprising the first 23 patients enrolled in this study [28]. This is also in line with a previous study proposing that circulating G-MDSCs may be more predominant in triple-negative breast cancer compared to other breast cancer subtypes [21]. ER-negative breast cancer has been associated with a stronger inflammatory response, where more inflammatory cytokines (e.g., IL-8 and IL-6) have been observed in tumors of patients with ER-negative breast cancer or in ER-negative breast cancer cell-lines [33, 34]. Pro-inflammatory stimulus is one important component of MDSC generation and accumulation [12]. The higher inflammatory activity associated with ER-negative breast cancer may consequently promote the accumulation of Mo-MDSCs.

Interestingly, the vast majority of patients with de novo MBC displayed high levels of Mo-MDSCs. De novo MBC differs from other breast cancer forms in terms of both characteristics and treatment strategies. Compared to early breast cancer, de novo MBC is a disease with systemic involvement already at first diagnosis. Though, unlike patients with distant recurrent $\mathrm{MBC}$, where the primary tumor had been resected at initial diagnosis, the primary tumor is still present in the subgroup of patients with de novo MBC. This implies that the primary tumor might impact the levels of Mo-MDSCs and that different biological mechanisms may be at play in patients with de novo MBC and distant recurrent MBC. Indeed, the levels of MDSCs decrease after removing the primary tumor in breast cancer mouse models and patients with MBC $[19,35]$. Studies have shown that cytokines such as GM-CSF, produced by tumor cells, may induce expansion of MDSCs $[12,13]$. It is also interesting to note that patients with early, localized, breast cancer generally display a modest enrichment in circulating MoMDSCs as compared to patients with locoregional recurrence or MBC [28]. The enrichment of Mo-MDSCs may thus be related to the metastatic or immunoregulatory switch during the transition to a more systemic disease [36]. This is supported by a study proposing that Mo-MDSCs themselves may induce a pro-metastatic phenotype of $4 \mathrm{~T} 1$ murine breast tumor cells [37].

The strong relationship between Mo-MDSCs and liverand bone metastases, as well as the observed associations with more CTCs, more metastatic sites, disease progression, and shorter progression-free and overall survival, indicate that Mo-MDSCs are related to a more aggressive metastatic disease and worse prognosis. This association was especially apparent in patients with distant recurrent MBC and is in line with previous studies where MDSCs in general are associated with clinical stage, metastatic burden and poor overall survival in breast cancer patients $[17,19]$. The potential correlation between Mo-MDSCs and CTCs was hitherto unexplored in breast cancer patients, although it has been proposed that MDSCs $\left(\mathrm{CD} 11 \mathrm{~b}^{+} \mathrm{CD} 33^{+} \mathrm{Lin}^{-} \mathrm{HLA}-\mathrm{DR}^{-}\right.$cells $)$ may correlate with CTCs [16]. Interestingly, CTCs have been suggested to preferably cluster together with leukocytes and that these clusters might be associated with worse prognosis in breast cancer patients [38]. The specific leukocyte population(s) was until recently uncharacterized. However, MDSC-like pro-tumor N2 neutrophils were recently shown to support the metastatic potential of CTCs in patients with metastatic breast cancer [39]. Whether also Mo-MDSCs, specifically, may play a role will be of interest to delineate in the future.

Tumor metastasis is a complex process and the outcome depends on both tumor properties and the host's response, accounting for the fact that only $0.01 \%$ of the malignant cells are estimated to succeed in establishing micro-metastases [40]. The process of establishing new metastatic sites is not fully understood, but has been proposed to involve formation of pre-metastatic niches. Intriguingly, several MDSC-associated mediators (such as S100A8/A9, MMP9 and IL-1 $\beta$ ) have been identified as important in establishment of pre-metastatic niches [40, 41]. Accordingly, mouse models of malignant melanoma have shown that both Mo-MDSCs and G-MDSCs are found in pre-metastatic sites weeks before the actual metastasis could be detected [41]. In fact, Mo-MDSC-derived cytokines have been proposed to facilitate adhesion of CTCs to the endothelium in the pre-metastatic organ [41]. How and why Mo-MDSCs would localize to pre-metastatic niches is not fully characterized, nor is it known whether this also occurs in human patients [42]. It is tempting to speculate that correlation between high levels of circulating Mo-MDSCs and specific metastatic sites, predominantly liver and bone, may be related to establishment of pre-metastatic niches by MDSCs [43]. This would also be in line with our hypothesis that enrichment of Mo-MDSCs is related to the metastatic and immunoregulatory switch in these patients. Further we show that high Mo-MDSC levels may be associated with impaired survival. This seems to be particularly relevant in patients with ER-positive MBC, a patient group with generally better prognosis compared to ER-negative MBC, implying that Mo-MDSC levels could be a prognostic factor in this subgroup of patients representing a more aggressive disease. Thus, although the inflammatory environment in ER-negative tumors promotes accumulation of Mo-MDSCs to a greater extent, the impact of Mo-MDSCs 
on survival may be limited due to the overall worse prognosis in this subgroup of patients.

Immune therapies are currently revolutionizing the field of oncology and check point inhibitors are being implemented as systemic therapies for different tumor types including breast cancer $[44,45]$. For MBC the PD-L1 inhibitor atezolizumab was recently shown to improve PFS and OS in patients with metastatic TNBC [46]. Mo-MDSCs are cells with capability to modulate the immune response in MBC patients and could hence be a potential target for immune therapies in the future. Our study is limited by the sample size, and some analyses thus fail to reach statistical significance. Despite this, strong trends are observed shedding light on the clinical impact of systemic Mo-MDSCs in breast cancer patients. An extended knowledge of the systemic immune response is, therefore, extremely relevant in understanding what patients will benefit most from immune therapies.

Acknowledgements Open access funding provided by Lund University. The authors thank research nurses Anette Ahlin-Gullers and Jessica Åkesson for preparation of blood samples from breast cancer patients.

Author contributions $\mathrm{CB}, \mathrm{KL}$ and $\mathrm{AML}$ were responsible for conception and design of the study. $\mathrm{CB}, \mathrm{AR}$ and $\mathrm{AML}$ were responsible for acquisition, analysis and interpretation of data and for drafting and reviewing the manuscript. $\mathrm{CB}$ and $\mathrm{MM}$ performed and analyzed the experiments. KL and MM were involved in reviewing the manuscript. All authors read and approved the final version of the manuscript.

Funding This work was supported by the Swedish Cancer Foundation, Vetenskapsrådet, Malmö Allmänna Sjukhus Cancer research foundation, Gunnar Nilssons Cancer Foundation, Ollie och Elof Ericssons Foundation, Governmental funding of Clinical Research within the National Health Service (ALF), the Gyllenstiernska Krapperups foundation and the Swedish Society for Medical Research (SSMF).

\section{Compliance with ethical standards}

Conflict of interest The authors declare no conflict of interests.

Ethical approval The present study is a sub-study of the prospective observational CTC-MBC study (ClinicalTrials.gov NCT01322893) including 156 women with newly diagnosed MBC (Ethical permit number: Dnr 2010/135, Dnr 2010/477 and Dnr 2011/748 for the CTCMBC study as well as the present sub-study). Ethical permit number for anonymous healthy blood donors: Dnr 2014/669. All work has been approved by the Regional Ethics Committee in Lund and been performed in accordance with the Declaration of Helsinki.

Informed consent All included patients and healthy blood donors signed a written informed consent, for research use of specimens and publication of data, at the time of inclusion in the clinical trial or when donating blood for clinical use, respectively.

Open Access This article is licensed under a Creative Commons Attribution 4.0 International License, which permits use, sharing, adaptation, distribution and reproduction in any medium or format, as long as you give appropriate credit to the original author(s) and the source, provide a link to the Creative Commons licence, and indicate if changes were made. The images or other third party material in this article are included in the article's Creative Commons licence, unless indicated otherwise in a credit line to the material. If material is not included in the article's Creative Commons licence and your intended use is not permitted by statutory regulation or exceeds the permitted use, you will need to obtain permission directly from the copyright holder. To view a copy of this licence, visit http://creativecommons.org/licenses/by/4.0/.

\section{References}

1. Bray F, Ferlay J, Soerjomataram I, Siegel RL, Torre LA, Jemal A (2018) Global cancer statistics 2018: GLOBOCAN estimates of incidence and mortality worldwide for 36 cancers in 185 countries. CA 68:394-424. Doi: 10.3322/caac.21492.

2. SweBCG (2018) Swedish Breast Cancer Group national guidelines. Available from http://www.swebcg.se

3. Tryggvadottir L, Gislum M, Bray F, Klint A, Hakulinen T, Storm HH, Engholm G (2010) Trends in the survival of patients diagnosed with breast cancer in the Nordic countries 1964-2003 followed up to the end of 2006. Acta Oncol 49:624-631. https://doi. org/10.3109/02841860903575323

4. Cardoso F, Costa A, Senkus E et al (2017) 3rd ESO-ESMO International Consensus Guidelines for Advanced Breast Cancer (ABC 3). Ann Oncolog 28:16-33. https://doi.org/10.1093/annon c/mdw544

5. Kim R, Emi M, Tanabe K (2007) Cancer immunoediting from immune surveillance to immune escape. Immunology 121:1-14. https://doi.org/10.1111/j.1365-2567.2007.02587.x

6. Schreiber RD, Old LJ, Smyth MJ (2011) Cancer immunoediting: integrating immunity's roles in cancer suppression and promotion. Science 331:1565-1570. https://doi.org/10.1126/science.1203486

7. Hanahan D, Weinberg RA (2011) Hallmarks of cancer: the next generation. Cell 144:646-674. https://doi.org/10.1016/j. cell.2011.02.013

8. Grivennikov SI, Greten FR, Karin M (2010) Immunity, inflammation, and cancer. Cell 140:883-899. https://doi.org/10.1016/j. cell.2010.01.025

9. Gabrilovich DI (2017) Myeloid-derived suppressor cells. Cancer Immunol Res 5:3-8. https://doi.org/10.1158/2326-6066. CIR-16-0297

10. Poschke I, Kiessling R (2012) On the armament and appearances of human myeloid-derived suppressor cells. Clin Immunol 144:250-268. https://doi.org/10.1016/j.clim.2012.06.003

11. Cuenca AG, Delano MJ, Kelly-Scumpia KM, Moreno C, Scumpia PO, Laface DM, Heyworth PG, Efron PA, Moldawer LL (2011) A paradoxical role for myeloid-derived suppressor cells in sepsis and trauma. Mol Med 17:281-292. https://doi.org/10.2119/molme d. 2010.00178

12. Millrud CR, Bergenfelz C, Leandersson K (2017) On the origin of myeloid-derived suppressor cells. Oncotarget 8:3649-3665. https ://doi.org/10.18632/oncotarget.12278

13. Talmadge JE, Gabrilovich DI (2013) History of myeloid-derived suppressor cells. Nat Rev Cancer 13:739-752. https://doi. org/10.1038/nrc3581

14. Gabrilovich DI, Nagaraj S (2009) Myeloid-derived suppressor cells as regulators of the immune system. Nat Rev Immunol 9:162-174. https://doi.org/10.1038/nri2506

15. Wang PF, Song SY, Wang TJ, Ji WJ, Li SW, Liu N, Yan CX (2018) Prognostic role of pretreatment circulating MDSCs in patients with solid malignancies: a meta-analysis of 40 studies. Oncoimmunology 7:e1494113. https://doi.org/10.1080/21624 02X.2018.1494113 
16. Markowitz J, Wesolowski R, Papenfuss T, Brooks TR, Carson WE 3rd (2013) Myeloid-derived suppressor cells in breast cancer. Breast Cancer Res Treat 140:13-21. https://doi.org/10.1007/s1054 9-013-2618-7

17. Diaz-Montero CM, Salem ML, Nishimura MI, Garrett-Mayer E, Cole DJ, Montero AJ (2009) Increased circulating myeloidderived suppressor cells correlate with clinical cancer stage, metastatic tumor burden, and doxorubicin-cyclophosphamide chemotherapy. Cancer Immunol Immunother 58:49-59. https:// doi.org/10.1007/s00262-008-0523-4

18. Foulds GA, Vadakekolathu J, Abdel-Fatah TMA et al (2018) Immune-phenotyping and transcriptomic profiling of peripheral blood mononuclear cells from patients with breast cancer: identification of a 3 gene signature which predicts relapse of triple negative breast cancer. Front Immunol 9:2028. https://doi.org/10.3389/ fimmu.2018.02028

19. Gonda K, Shibata M, Ohtake T, Matsumoto Y, Tachibana K, Abe N, Ohto H, Sakurai K, Takenoshita S (2017) Myeloid-derived suppressor cells are increased and correlated with type 2 immune responses, malnutrition, inflammation, and poor prognosis in patients with breast cancer. Oncol Lett 14:1766-1774. https:// doi.org/10.3892/ol.2017.6305

20. Solito S, Falisi E, Diaz-Montero CM et al (2011) A human promyelocytic-like population is responsible for the immune suppression mediated by myeloid-derived suppressor cells. Blood 118:2254-2265. https://doi.org/10.1182/blood-2010-12-325753

21. Wesolowski R, Duggan MC, Stiff A et al (2017) Circulating myeloid-derived suppressor cells increase in patients undergoing neoadjuvant chemotherapy for breast cancer. Cancer Immunol Immunother 66:1437-1447. https://doi.org/10.1007/s00262-017-2038-3

22. Poschke I, Mougiakakos D, Hansson J, Masucci GV, Kiessling $\mathrm{R}$ (2010) Immature immunosuppressive CD14+HLA-DR-/low cells in melanoma patients are Stat3hi and overexpress CD80, CD83, and DC-sign. Cancer Res 70:4335-4345. https://doi. org/10.1158/0008-5472.CAN-09-37670008-5472.CAN-09-3767 [pii]

23. Filipazzi P, Valenti R, Huber V et al (2007) Identification of a new subset of myeloid suppressor cells in peripheral blood of melanoma patients with modulation by a granulocytemacrophage colony-stimulation factor-based antitumor vaccine. J Clin Oncol 25:2546-2553. https://doi.org/10.1200/ JCO.2006.08.582925/18/2546 [pii]

24. Vuk-Pavlovic S, Bulur PA, Lin Y, Qin R, Szumlanski CL, Zhao X, Dietz AB (2010) Immunosuppressive CD14+HLA-DRlow/monocytes in prostate cancer. Prostate 70:443-455. https://doi. org/10.1002/pros. 21078

25. Gustafson MP, Lin Y, New KC, Bulur PA, O’Neill BP, Gastineau DA, Dietz AB (2010) Systemic immune suppression in glioblastoma: the interplay between CD14+HLA-DRlo/neg monocytes, tumor factors, and dexamethasone. Neuro-Oncol 12:631-644. https://doi.org/10.1093/neuonc/noq001

26. Lin Y, Gustafson MP, Bulur PA, Gastineau DA, Witzig TE, Dietz AB (2011) Immunosuppressive CD14+HLA-DR(low)/- monocytes in B-cell non-Hodgkin lymphoma. Blood 117:872-881. https://doi.org/10.1182/blood-2010-05-283820

27. Yuan XK, Zhao XK, Xia YC, Zhu X, Xiao P (2011) Increased circulating immunosuppressive CD14(+)HLA-DR(-/low) cells correlate with clinical cancer stage and pathological grade in patients with bladder carcinoma. J Int Med Res 39:1381-1391

28. Bergenfelz C, Larsson AM, von Stedingk K et al (2015) Systemic monocytic-MDSCs are generated from monocytes and correlate with disease progression in breast cancer patients. PLoS ONE 10:e0127028. https://doi.org/10.1371/journal.pone.0127028

29. Larsson AM, Jansson S, Bendahl PO, Levin Tykjaer Jorgensen C, Loman N, Graffman C, Lundgren L, Aaltonen K, Ryden L (2018) Longitudinal enumeration and cluster evaluation of circulating tumor cells improve prognostication for patients with newly diagnosed metastatic breast cancer in a prospective observational trial. Breast Cancer Res 20:48. https://doi.org/10.1186/s1305 8-018-0976-0

30. Eisenhauer EA, Therasse P, Bogaerts J et al (2009) New response evaluation criteria in solid tumours: revised RECIST guideline (version 1.1). Eur J Cancer 45:228-247. https://doi.org/10.1016/j. ejca.2008.10.026

31. Cristofanilli M, Budd GT, Ellis MJ et al (2004) Circulating tumor cells, disease progression, and survival in metastatic breast cancer. $\mathrm{N}$ Engl J Med 351:781-791. https://doi.org/10.1056/NEJMoa040766

32. Allard WJ, Matera J, Miller MC, Repollet M, Connelly MC, Rao C, Tibbe AG, Uhr JW, Terstappen LW (2004) Tumor cells circulate in the peripheral blood of all major carcinomas but not in healthy subjects or patients with nonmalignant diseases. Clin Cancer Res 10:6897-6904. https://doi.org/10.1158/1078-0432. CCR-04-0378

33. Chavey C, Bibeau F, Gourgou-Bourgade S, Burlinchon S, Boissiere F, Laune D, Roques S, Lazennec G (2007) Oestrogen receptor negative breast cancers exhibit high cytokine content. Breast Cancer Res 9:R15. https://doi.org/10.1186/bcr1648

34. Mehmeti M, Allaoui R, Bergenfelz C, Saal LH, Ethier SP, Johansson ME, Jirstrom K, Leandersson K (2015) Expression of functional toll like receptor 4 in estrogen receptor/progesterone receptor-negative breast cancer. Breast Cancer Res 17:130. https ://doi.org/10.1186/s13058-015-0640-x

35. Rashid OM, Nagahashi M, Ramachandran S, Graham L, Yamada A, Spiegel S, Bear HD, Takabe K (2013) Resection of the primary tumor improves survival in metastatic breast cancer by reducing overall tumor burden. Surgery 153:771-778. https://doi. org/10.1016/j.surg.2013.02.002

36. Gil Del Alcazar CR, Huh SJ, Ekram MB et al (2017) Immune escape in breast cancer during in situ to invasive carcinoma transition. Cancer Discov 7:1098-1115. https://doi.org/10.1158/21598290.CD-17-0222

37. Ouzounova M, Lee E, Piranlioglu R et al (2017) Monocytic and granulocytic myeloid derived suppressor cells differentially regulate spatiotemporal tumour plasticity during metastatic cascade. Nat Commun 8:14979. https://doi.org/10.1038/ncomms14979

38. Jansson S, Bendahl PO, Larsson AM, Aaltonen KE, Ryden L (2016) Prognostic impact of circulating tumor cell apoptosis and clusters in serial blood samples from patients with metastatic breast cancer in a prospective observational cohort. BMC Cancer 16:433. https://doi.org/10.1186/s12885-016-2406-y

39. Szczerba BM, Castro-Giner F, Vetter M et al (2019) Neutrophils escort circulating tumour cells to enable cell cycle progression. Nature 566:553-557. https://doi.org/10.1038/s41586-019-0915-y

40. Joyce JA, Pollard JW (2009) Microenvironmental regulation of metastasis. Nat Rev Cancer 9:239-252. https://doi.org/10.1038/ $\operatorname{nrc} 2618$

41. Shi H, Zhang J, Han X, Li H, Xie M, Sun Y, Liu W, Ba X, Zeng X (2017) Recruited monocytic myeloid-derived suppressor cells promote the arrest of tumor cells in the premetastatic niche through an IL-1beta-mediated increase in E-selectin expression. Int J Cancer 140:1370-1383. https://doi.org/10.1002/ijc.30538

42. Pastaki Khoshbin A, Eskian M, Keshavarz-Fathi M, Rezaei N (2018) Roles of myeloid-derived suppressor cells in cancer metastasis: immunosuppression and beyond. Arch Immunol Ther Exp (Warsz). https://doi.org/10.1007/s00005-018-0531-9

43. Ursini-Siegel J, Siegel PM (2016) The influence of the pre-metastatic niche on breast cancer metastasis. Cancer Lett 380:281-288. https://doi.org/10.1016/j.canlet.2015.11.009

44. Solinas C, Gombos A, Latifyan S, Piccart-Gebhart M, Kok M, Buisseret L (2017) Targeting immune checkpoints in breast cancer: an update of early results. ESMO Open 2:e000255. https:// doi.org/10.1136/esmoopen-2017-000255 
45. Katz H, Alsharedi M (2017) Immunotherapy in triple-negative breast cancer. Med Oncol 35:13. https://doi.org/10.1007/s1203 2-017-1071-6

46. Schmid P, Adams S, Rugo HS et al (2018) Atezolizumab and nab-paclitaxel in advanced triple-negative breast cancer. N Engl J Med 379:2108-2121. https://doi.org/10.1056/NEJMoa1809615
Publisher's Note Springer Nature remains neutral with regard to jurisdictional claims in published maps and institutional affiliations. 